\title{
A Logical Characterization of Efficiency Preorders
}

\author{
Neelesh Korade ${ }^{1}$ and S. Arun-Kumar ${ }^{2 \star}$ \\ 1 Persistent Systems Private Limited, "Bhageerath", 402, Senapati Bapat Road Pune 411016, \\ India. email: neelesh_korade@persistent.co.in \\ 2 Department of Computer Science and Engineering, Indian Institute of Technology, Delhi, \\ Hauz Khas, New Delhi 110016, India. email: sakecse.iitd.ernet. in
}

\begin{abstract}
In this paper we present logical characterizations of two preorders, within the framework of Hennessy-Milner Logics. The two preorders (loosely termed bisimulation-based efficiency preorders) are on processes represented as labelled transition systems. The characterizations are particularly interesting as they explore preorders lying between strong and weak bisimilarity, guided by a principle of containment which is explained in the Introduction. Even though the proofs of the characterizations use standard methods, there are various subtleties introduced by the nature of the preorders and the logical operators needed to characterize them. The authors have not previously encountered the use of such operators in such simple logics.
\end{abstract}

Keywords Concurrency, transition systems, bisimulation, efficiency preorders, process efficiency, Hennessy-Milner Logic.

\section{Introduction}

In [5] a modal logic for reasoning about labelled transition systems was first defined which characterized the notions of simulations, strong and weak bisimulations. Subsequently the logic has been extended in various ways to include the modal $\mu$-calculus and various behavioural equivalences and preordering relations on labelled transition systems. For a comprehensive account, the reader is referred to [12].

In all such formulations, a process is identified by the set of formulas of a logic that it satisfies. Given a behavioural equivalence relation on processes, a logical language $L$ characterizes this equivalence relation precisely when two equivalent processes satisfy the same set of formulas of the logic.

Formally therefore, if $\mathbb{P}$ is a set of processes and $L$ is a logic then we may identify each process $p \in \mathbb{P}$ with the set of formulas of $L$ that it satisfies, i.e. $L(p)=\{\phi \in L \mid p \vDash \phi\}$ denotes the set of formulas of $L$ that $p$ satisfies. A behavioural equivalence $\cong$ on $\mathbb{P}$ is characterized by $L$ whenever $p \cong q$ iff $L(p)=L(q)$. Similarly a behavioural preorder $\lesssim$ on $\mathbb{P}$ is characterized by $L$ whenever $p \lesssim q$ iff $L(p) \subseteq L(q)$ or $p \lesssim q$ iff $L(q) \subseteq L(p)$.

\footnotetext{
^ corresponding author
} 
Van Glabeek [14] has used precisely such formulations to characterize the various behavioural relations on concrete processes. In several instances, it has been shown that one behavioural relation $R$ is coarser than another $S$, by showing that a less expressive logic characterizes $R$ or that a more expressive logic characterizes $S$.

Such a formulation may actually be traced back to [5]. In it the authors present two modal logics - the stronger or more expressive one (subsequently referred to in the literature as Hennessy-Milner Logic or HML) characterizing strong bisimulation equivalence and the weaker or less expressive one (referred to by Stirling [12] as Observable Hennessy-Milner Logic or OHML) characterizing observational equivalence. The paper [5] also showed the characterization of trace equivalence and simulation equivalence in terms of sub-logics of HML, thus establishing that both trace equivalence and the simulation equivalence are coarser than bisimulation. The authors actually showed that for image-finite processes, HML with finitary conjunctions captured strong bisimilarity whereas HML without negation characterized simulation equivalence. Trace equivalence was characterized by removing both negation and conjunction from the logic.

In this paper we provide such logical characterizations for behavioural relations that lie strictly between strong and weak bisimilarity by defining modified Hennessy-Milner Logics for the purpose. We use the following properties as guiding principles in the design of the logics.

1. For any behavioural preorder $\leq$ and logical language $L$ characterizing $\leq, p \leq q$ iff $L(p) \subseteq L(q)$. It then follows that the kernel of the preorder is an equivalence relation and is characterized by equality on sets of satisfying formulae. That is, $p \leq q$ and $q \leq p$ if and only if $L(p)=L(q)$.

2. Given preorders $\leq_{1}$, and $\leq_{2}$ characterized respectively by logics $L_{1}$ and $L_{2}, \leq_{1} \subset$ $\leq_{2}$ iff $L_{1} \prec L_{2}$ where $L_{1} \prec L_{2}$ denotes that $L_{1}$ is more expressive than $L_{2}$ (and hence can allow for finer distinctions to be made).

While adhering to these principles, it may be pointed out that so far the spectrum of behavioural relations that lie strictly between strong and weak bisimilarity has not really been explored in the literature. This includes some preorders defined by Milner and others ([11], [10], [3], [2]). In this paper we provide a characterization of the preorders defined in [3] and [2]. As we will show, the characterization of these preorders requires reasoning about linear orders within logics whose expressive power lies strictly between HML and OHML.

Efficiency-based preorders have been of interest to various people since they were first introduced in [1]. Several other authors have worked on obtaining similar preorders within the framework of extensionality (see [13], [6], [7], [8], [4]). Some of these works are based on extent theories such as testing and bisimulation in process algebra and Petri nets.

The paper is organized as follows. In the next section we review HML and OHML and show the characterizations of strong and weak bisimilarity respectively. Section 3 presents the logical characterization of the elaboration preorder [3] using an appropriately modified OHML. In this section we also highlight the principal differences 
between the elaboration preorder and weak bisimilarity and hence the need for a more expressive logic than OHML to characterize elaboration. In section 4, we present the characterization of the efficiency preorder relation. Though the proofs in these sections are standard the operators introduced to characterize the two preorders are not standard. Section 5 is the conclusion and highlights further properties of the new modal operators that have been introduced to enrich OHML so as to characterize these preorders.

\section{Modal Characterizations of Strong and Weak Bisimilarity}

Here we review Hennessy-Milner Logic (HML) and Observable Hennessy-Milner Logic (OHML) and show how they characterize strong and weak bisimilarity respectively.

Let $V$ be a set of visible actions, $\tau \notin V$ a distinguished invisible action and Act $=V \cup\{\tau\}$ the set of actions. A labelled transition system $($ LTS $)$ is a 3-tuple $\langle\mathbb{P}, A c t, \longrightarrow\rangle$, where $\mathbb{P}$ is a set of process states or processes and $\longrightarrow \subseteq \mathbb{P} \times A c t \times \mathbb{P}$ is the transition relation. We use the notation $p \stackrel{a}{\longrightarrow} q$ to denote $(p, a, q) \in \longrightarrow$ and refer to $q$ as a strong a-derivative of $p$.

\subsection{Strong Bisimulation}

Definition 1. A binary relation $R \subseteq \mathbb{P} \times \mathbb{P}$ is a strong simulation (SS) if for every $\langle p, q\rangle \in R$ and $a \in A c t$ :

$$
p \stackrel{a}{\longrightarrow} p^{\prime} \Longrightarrow \exists q^{\prime}: q \stackrel{a}{\longrightarrow} q^{\prime} \wedge p^{\prime} R q^{\prime}
$$

It is a strong bisimulation (SB) if both $R$ and $R^{-1}$ are strong simulations. We write $p \sim q$ if there exists a strong bisimulation $R$ such that $p R q$. The relation $\sim$ is called strong bisimilarity.

The relation $\sim$ is itself a strong bisimulation, and in fact, the largest one. In [9] the author gives a comprehensive account of the modal logic (HML) that characterizes strong bisimilarity. It is defined as follows.

Definition 2. The class $L_{S B}$ of strong bisimulation formulas over Act is given by the following grammar ( $I$ is an indexing set, not necessarily finite).

$$
\varphi::=<a>\varphi\left|\bigwedge_{i \in I} \varphi_{i}\right| \neg \varphi
$$

$t t \in L_{S B}$ where $t t \equiv \bigwedge_{i \in \emptyset} \varphi_{i}$. Similarly, ff $\in L_{S B}$ where $f f \equiv \neg t$.

Definition 3. The satisfaction relation $\vDash \subseteq \mathbb{P} \times L_{S B}$ is defined recursively as follows:

$$
-p \vDash t t \text { for all } p \in \mathbb{P} \text {. }
$$


- $p \vDash<a>\varphi$ for $a \in A c t$ if $\exists p^{\prime} \in \mathbb{P}: p \stackrel{a}{\longrightarrow} p^{\prime}$ and $p^{\prime} \vDash \varphi$.

$-p \vDash \bigwedge_{i \in I} \varphi_{i}$ if $p \vDash \varphi_{i}$ for all $i \in I$.

$-p \vDash \neg \varphi$ if $p \not \models \varphi$.

The set $S B(p)$ is defined as $S B(p)=\left\{\varphi \in L_{S B} \mid p \vDash \varphi\right\}$. We write $p \sqsubseteq S B q$ iff $S B(p) \subseteq$ $S B(q)$ and we write $p={ }_{S B} q$ iff $S B(p)=S B(q)$. The negation operator collapses the preorder $\sqsubseteq_{S B}$ to $=_{S B}$ as the following proposition shows.

Proposition 4 (Van Glabeek [14]). $p \sqsubseteq_{S B} q \Longleftrightarrow p={ }_{S B} q$.

Proof. If $\varphi \in S B(q)-S B(p)$ then $\neg \varphi \in S B(p)-S B(q)$. Hence, $p \sqsubseteq_{S B} q \Longleftrightarrow p=_{S B} q$.

Theorem 5 (Van Glabeek [14]). $p \sim q \Longleftrightarrow p=_{S B} q$.

Proof. $(\Longrightarrow)$ By induction on the structure of $\varphi$. Since $p \sim q$, there exists a strong bisimulation $R$ such that $p R q$.

- Let $p \vDash<a>\varphi$. Then there exists a $p^{\prime} \in \mathbb{P}$ with $p \stackrel{a}{\longrightarrow} p^{\prime}$ and $p^{\prime} \vDash \varphi$. Since $p R q$, there must be a $q^{\prime} \in \mathbb{P}$ with $q \stackrel{a}{\longrightarrow} q^{\prime}$ and $p^{\prime} R q^{\prime}$. So by induction $q^{\prime} \vDash \varphi$ and hence $q \vDash<a>\varphi$.

By symmetry, one also obtains $q \vDash<a>\varphi \Longrightarrow p \vDash<a>\varphi$.

- $p \vDash \bigwedge_{i \in I} \varphi_{i} \Longleftrightarrow \forall i \in I\left(p \vDash \varphi_{i}\right) \stackrel{\text { induction }}{\Longleftrightarrow} \forall i \in I\left(q \vDash \varphi_{i}\right) \Longleftrightarrow q \vDash \bigwedge_{i \in I} \varphi_{i}$.

- $p \vDash \neg \varphi \Longleftrightarrow p \not \models \varphi \stackrel{\text { induction }}{\Longleftrightarrow} q \not \models \varphi \Longleftrightarrow q \vDash \neg \varphi$.

( $\Longleftrightarrow$ ) To prove that $p={ }_{S B} q \Longrightarrow p \sim q$, it suffices to establish that $=_{S B}$ is a strong bisimulation. If however, we show that $\bigsqcup_{S B}$ is a strong simulation, then proposition 4 implies that $=_{S B}=\sqsubseteq_{S B}=\sqsubseteq_{S B}^{-1}$ is a strong bisimulation. We proceed to show that $\sqsubseteq_{S B}$ is a strong simulation.

Suppose, $p \sqsubseteq_{S B} q$ and $p \stackrel{a}{\longrightarrow} p^{\prime}$. Then $p \vDash<a>t t$ and $p \sqsubseteq_{S B} q$ implies $q$ has at least one strong $a$-derivative. We have to show that $\exists q^{\prime} \in \mathbb{P}$ with $q \stackrel{a}{\longrightarrow} q^{\prime}$ and $p^{\prime} \sqsubseteq_{S B} q^{\prime}$. Let

$$
Q=\left\{q^{\prime \prime} \in \mathbb{P} \mid q \stackrel{a}{\longrightarrow} q^{\prime \prime} \wedge p^{\prime} \nsubseteq_{S B} q^{\prime \prime}\right\}
$$

For every $q^{\prime \prime} \in Q$ there is a formula $\varphi_{q^{\prime \prime}} \in S B\left(p^{\prime}\right)-S B\left(q^{\prime \prime}\right)$. Now $<a>\bigwedge_{q^{\prime \prime} \in Q} \varphi_{q^{\prime \prime}} \in$ $S B(p) \subseteq S B(q)$. Therefore there must be a $q^{\prime} \in \mathbb{P}$ with $q \stackrel{a}{\longrightarrow} q^{\prime}$ and $q^{\prime} \vDash \bigwedge_{q^{\prime \prime} \in Q} \varphi_{q^{\prime \prime}}$, which implies $q^{\prime} \notin Q$.

With negation in the logic, we may define the duals of the operators in $L_{S B}$ as derived ones.

$$
[a] \varphi \equiv \neg<a>\neg \varphi \quad \bigvee_{i \in I} \varphi_{i} \equiv \neg \bigwedge_{i \in I} \neg \varphi_{i}
$$

We may then define a negation-free language $L_{S B^{\prime}}$. 
Definition 6. The class $L_{S B^{\prime}}$ of negation-free strong bisimulation formulas over Act is given by the following grammar

$$
\varphi::=<a>\varphi\left|\bigwedge_{i \in I} \varphi_{i}\right|[a] \varphi \mid \bigvee_{i \in I} \varphi_{i}
$$

with $t t \equiv \bigwedge_{i \in \emptyset} \varphi_{i}$ and $f f \equiv \bigvee_{i \in \emptyset} \varphi_{i}$

Definition 7. The satisfaction relation $\vDash \subseteq \mathbb{P} \times L_{S B^{\prime}}$ is defined recursively as in the case of $L_{S B}$ with the clause for negation being replaced by the following clauses.

- $p \vDash f f$ for no $p \in \mathbb{P}$.

- $p \vDash[a] \varphi$ for $a \in A c t$ if $\forall p^{\prime} \in \mathbb{P}: p \stackrel{a}{\longrightarrow} p^{\prime} \Longrightarrow p^{\prime} \vDash \varphi$.

- $p \vDash \bigvee_{i \in I} \varphi_{i}$ if $p \vDash \varphi_{i}$ for some $i \in I$.

It is clear that $\neg \neg \varphi$ is equivalent to $\varphi$. It may be shown (refer [12]) that $L_{S B}$ and $L_{S B^{\prime}}$ are expressively equivalent and characterize the same equivalence.

\subsection{Weak Bisimulation}

We may define several other derived operators. Some of the relevant ones are given below.

$$
\begin{aligned}
& <a>^{0} \varphi \equiv \varphi \\
& <>>\varphi \equiv \bigvee_{m \geq 0}<\tau>^{m} \varphi \\
& \text { [[]] } \varphi \equiv \neg<<>>\neg \varphi \\
& \begin{array}{l}
<a>^{m+1} \varphi \equiv<a><a>^{m} \varphi, m \in \mathbb{N} \\
<<a>>\varphi \equiv<<><a><<>>\varphi \\
{[[a]] \varphi \quad \equiv[[]][a][[]] \varphi}
\end{array}
\end{aligned}
$$

The last four operators correspond to the weak transition relation $\Longrightarrow$ which is the smallest relation on processes such that

- $p \Longrightarrow p$ for all processes $p$, and

$-p \stackrel{\tau}{\longrightarrow} q$ and $q \Longrightarrow r$ implies $p \Longrightarrow r$

Further for any $a \in A c t$,

$-p \stackrel{a}{\Longrightarrow} q$ if $p \Longrightarrow \stackrel{a}{\Longrightarrow} \Longrightarrow$, and $q$ is called a weak a-derivative or simply an $a$ derivative of $p$.

$-p \stackrel{\hat{a}}{\Longrightarrow} q$ denotes $p \Longrightarrow q$ if $a=\tau$ and $p \stackrel{a}{\Longrightarrow} q$ otherwise.

$-\langle\langle\hat{a}\rangle\rangle$ denotes $<<>>$ if $a=\tau$ and $\langle<a\rangle>$ otherwise.

Definition 8. A binary relation $R \subseteq \mathbb{P} \times \mathbb{P}$ is a weak simulation if for every $\langle p, q\rangle \in R$ and $a \in A c t$,

$$
p \stackrel{a}{\longrightarrow} p^{\prime} \Longrightarrow \exists q^{\prime}: q \stackrel{\hat{a}}{\Longrightarrow} q^{\prime} \wedge p^{\prime} R q^{\prime}
$$

$R$ is a weak bisimulation (WB) if both $R$ and $R^{-1}$ are weak simulations. We write $p \approx q$ if there exists a weak bisimulation $R$ such that $p R q$. 
Definition 9. The class $L_{W B}$ of weak bisimulation formulae over Act is defined by the following BNF.

$$
\varphi::=<\alpha \alpha>\varphi\left|\bigwedge_{i \in I} \varphi_{i}\right| \neg \varphi
$$

where $\alpha \in V$.

Note that in the light of our discussion on derived operators, $L_{W B}$ is entirely contained in $L_{S B}$. It is also less expressive in the sense that it cannot express properties relating to the number of invisible moves a process might be able to make.

Definition 10. The satisfaction relation $\vDash \subseteq \mathbb{P} \times L_{W B}$ is defined recursively by:

$-p \vDash t t$ for all $p \in \mathbb{P}$.

- $p \vDash<<\alpha>\rangle$ for $\alpha \in V$ if $\exists p^{\prime}: p \stackrel{\alpha}{\Longrightarrow} p^{\prime} \wedge p^{\prime} \vDash \varphi$

$-p \vDash \bigwedge_{i \in I} \varphi_{i}$ if $p \vDash \varphi_{i}$ for all $i \in I$.

$-p \vDash \neg \varphi$ if $p \not \models \varphi$.

The set of all formulae that a process $p$ satisfies is defined as $W B(p)=\left\{\varphi \in L_{W B} \mid p \vDash\right.$ $\varphi\}$. We write $p \sqsubseteq W B q$ iff $W B(p) \subseteq W B(q)$ and we write $p={ }_{W B} q$ iff $W B(p)=W B(q)$. The proofs that $p \sqsubseteq_{W B} q$ implies $p=_{W B} q$ and that $p \approx q$ iff $p=_{W B} q$ (i.e. that $L_{W B}$ characterizes weak bisimilarity) are similar to the corresponding proofs for strong bisimilarity and hence are omitted. It is also possible to define a negation-free logic $L_{W B^{\prime}}$ (analogous to $L_{S B^{\prime}}$ ) which characterizes weak bisimilarity.

\section{Elaboration}

In this section we give a logical characterization of the elaboration preorder defined in [3]. This logic builds upon $L_{W B}$ and exhibits controlled use of counting.

Definition 11. A binary relation $R \subseteq \mathbb{P} \times \mathbb{P}$ is an elaboration iff for every $\langle p, q\rangle \in R$ the following conditions hold for every action $a \in$ Act.

$$
\begin{gathered}
p \stackrel{a}{\longrightarrow} p^{\prime} \Longrightarrow \exists q^{\prime}: q \stackrel{\hat{a}}{\Longrightarrow} q^{\prime} \wedge p^{\prime} R q^{\prime} \\
q \stackrel{a}{\longrightarrow} q^{\prime} \Longrightarrow \exists p^{\prime}: p \stackrel{a}{\Longrightarrow} p^{\prime} \wedge p^{\prime} R q^{\prime}
\end{gathered}
$$

We write $p \lesssim q$ if there exists an elaboration $R$ such that $p R q$.

Loosely speaking, if $p \lesssim q$, then $p \approx q$ and for every execution sequence of $p$, there exists a possibly shorter execution sequence of $q$ which exhibits the same visible behaviour. In [3] the authors illustrate this feature with a small example. They also show in the equational axiomatization of the corresponding precongruence (and congruence) for finite CCS processes, that it differs from that of observational congruence in just one equation. Whereas observational congruence satisfies the equation $a . \tau \cdot p=a . p$, in

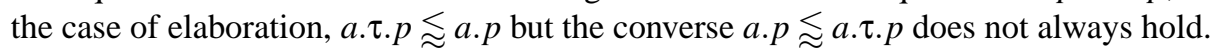


In that sense the precongruence is closer to observational congruence than any other relation defined so far in the literature.

It is easy to show from the above definition that every elaboration is a weak bisimulation. In particular, the only difference from that of weak bisimulation is in clause (2), where the $\stackrel{\hat{a}}{\Longrightarrow}$ is replaced by $\stackrel{a}{\Longrightarrow}$. We state without proof, a result (see [3]) that we use in the proof of lemma 19.

Lemma 12. If $R \subseteq \mathbb{P} \times \mathbb{P}$ is an elaboration then for every $\langle p, q\rangle \in R$ the following conditions hold for every action $a \in$ Act.

$$
\begin{gathered}
p \stackrel{a}{\Longrightarrow} p^{\prime} \Longrightarrow \exists q^{\prime}: q \stackrel{\hat{a}}{\Longrightarrow} q^{\prime} \wedge p^{\prime} R q^{\prime} \\
q \stackrel{a}{\Longrightarrow} q^{\prime} \Longrightarrow \exists p^{\prime}: p \stackrel{a}{\Longrightarrow} p^{\prime} \wedge p^{\prime} R q^{\prime}
\end{gathered}
$$

From lemma 12 it is clear that any HML-style characterization that is in accordance with the principles laid out in section 1 would enrich observable HML slightly in order to characterize the preorder. We define the following two-level grammar, whose lower level (defined by the meta-variable $\varphi$ ) is $L_{W B}$. The higher level (defined by the metavariable $\pi$ ) expresses a certain weak form of counting. It introduces a new operator $\varepsilon^{k}$, (where $k$ is a positive integer) but does not permit negation to precede any occurrence of $\varepsilon^{k}$. The operator $\langle<\hat{a}\rangle>$ excludes $\langle<\tau\rangle>$. Where counting of $\tau$ actions is not important, the lack of negation is compensated by the derived operators $[[\ldots]]$ and $\bigvee$.

Definition 13. The class $L_{E}$ of Elaboration formulae over Act is given by the following two-level grammar, where $\alpha \in V$ and $a \in A$.

$$
\begin{aligned}
& \varphi::=\left\langle<\alpha>\varphi\left|\bigwedge_{i \in I} \varphi_{i}\right| \neg \varphi\right. \\
& \pi::=\varphi|<<\hat{a} \gg>\pi| \varepsilon^{k} \pi|[[a]] \pi| \bigwedge_{i \in I} \pi_{i} \mid \bigvee_{i \in I} \pi_{i}
\end{aligned}
$$

Definition 14. The satisfaction relation $\vDash \subseteq \mathbb{P} \times L_{E}$ is defined recursively in a manner that should be obvious by now for all operators drawn from $L_{W B}$. So we restrict ourselves to the definitions of the operators $\langle\langle\hat{a}\rangle\rangle, \varepsilon^{k}$ and $[[a]]$ respectively.

- $p \vDash<<\hat{a}>>\pi$ for $a \in A c t$, if $\exists p^{\prime} \in \mathbb{P}: p \stackrel{\hat{a}}{\Longrightarrow} p^{\prime} \wedge p^{\prime} \vDash \pi$.

- $p \vDash \varepsilon^{k} \pi$ for $k>0$, if $\forall p^{\prime} \in \mathbb{P}: p \stackrel{\tau^{j}}{\longrightarrow} p^{\prime} \wedge p^{\prime} \vDash \pi \Longrightarrow j<k$

$-p \vDash[[a]] \pi$ for $a \in$ Act if for all $p^{\prime} \in \mathbb{P}: p \stackrel{a}{\Longrightarrow} p^{\prime} \Longrightarrow p^{\prime} \vDash \pi$.

$p \stackrel{\tau^{j}}{\longrightarrow} p^{\prime}$ denotes that $p$ may evolve to $p^{\prime}$ after performing $j$ consecutive invisible actions. $E(p)=\left\{\pi \in L_{E} \mid p \vDash \pi\right\}$ and $E_{W B}(p)=\left\{\varphi \in L_{W B} \mid p \vDash \varphi\right\}$. We write $p \sqsubseteq_{E} q$ iff $E(p) \subseteq$ $E(q)$ and $p={ }_{E} q$ iff $E(p)=E(q)$. Similarly, $p \sqsubseteq E_{W B} q$ iff $E_{W B}(p) \subseteq E_{W B}(q)$ and $p=E_{W B}$ $q$ iff $E_{W B}(p)=E_{W B}(q)$.

To gain a deeper understanding, we give below some examples that illustrate the expressive power of $L_{E}$. 


\section{Example 15.}

- The statement " $p$ is stable" i.e. $p$ cannot perform a $\tau$ action, may be expresed as $\varepsilon^{1} t t$. More generally, $p \vDash \varepsilon^{k} t t$ if and only if $p$ may perform no more than $(k-1)$ consecutive $\tau$ actions.

- The statement " $p$ converges" i.e. $p$ cannot perform an infinite sequence of $\tau$ actions, is expressed as $\bigvee_{k>0} \varepsilon^{k} t t$.

- The statement " $p$ diverges" is however not expressible in the language since $p$ diverges iff $p \not \models \bigvee_{k>0} \varepsilon^{k} t t$.

- The statement $p \vDash \varepsilon^{k} f f$ means that " $p$ can perform $\tau^{m}$ for all $m \geq k$ ". However, this statement does not necessarily imply that $p$ diverges, unless $p$ is also finitely branching.

- $\varepsilon^{j} \pi$ logically implies $\varepsilon^{k} \pi$ for all $0<j<k$.

- For any $j>0, \bigwedge_{k \geq j} \varepsilon^{k} \pi$ is logically equivalent to $\varepsilon^{j} \pi$.

- Statements such as " $p$ can do at least two consecutive $\tau$ actions" are not expressible since there is no operator which can express lower bounds on the number of consecutive $\tau$ actions ${ }^{1}$.

We now proceed to prove the characterization theorem. We begin with the following lemma which is clearly implied by the fact that every elaboration is a weak bisimulation and that $\sqsubseteq_{W B}$ and $=E_{W B}$ coincide.

Lemma 16. $p \lesssim q \Longrightarrow p==_{E_{W B}} q$ and hence $p \lesssim q \Longrightarrow p \sqsubseteq E_{W B} q$.

Theorem 17. The characterization. $p \lesssim q$ iff $p \sqsubseteq_{E} q$.

We split the proof of theorem 17 into two parts.

Lemma 18. $p \sqsubseteq_{E} q$ implies $p \lesssim q$.

Proof. We show that $\complement_{E}$ is an elaboration. Let $E(p) \subseteq E(q)$. We need to prove both parts (1) and (2) of definition 11.

Part (1). Consider $p \stackrel{a}{\longrightarrow} p^{\prime}$ where $a \in A c t$. Then $p \vDash<<\hat{a} \gg>\pi$ for any $\pi \in E\left(p^{\prime}\right)$ and $q \vDash<<\hat{a} \gg>\pi$ which implies $\exists q^{\prime}: q \stackrel{\hat{a}}{\Longrightarrow} q^{\prime} \wedge q^{\prime} \vDash \pi$. We need to show that $\exists q^{\prime}: q \stackrel{\hat{a}}{\Longrightarrow}$ $q^{\prime} \wedge E\left(p^{\prime}\right) \subseteq E\left(q^{\prime}\right)$.

Let

$$
Q=\left\{q^{\prime \prime} \mid q \stackrel{\hat{a}}{\Longrightarrow} q^{\prime \prime} \wedge E\left(p^{\prime}\right) \nsubseteq E\left(q^{\prime \prime}\right)\right\}
$$

Then for each $q^{\prime \prime} \in Q$, there exists a formula $\pi_{q^{\prime \prime}} \in E\left(p^{\prime}\right)-E\left(q^{\prime \prime}\right)$. This implies $p^{\prime} \vDash$ $\bigwedge_{q^{\prime \prime} \in Q} \pi_{q^{\prime \prime}}$. Hence $p \vDash \pi$ where $\pi=<<\hat{a} \gg>\bigwedge_{q^{\prime \prime} \in Q} \pi_{q^{\prime \prime}}$. That is, $\pi \in E(p) \subseteq E(q)$. Hence

\footnotetext{
${ }^{1}$ To be able to express such statements, would require either the power of negation or the dual of $\varepsilon^{k}$. But allowing such operators would make the logic equivalent to $L_{S B}$, something we wish to avoid.
} 
$\exists q^{\prime}: q \stackrel{\hat{a}}{\Longrightarrow} q^{\prime} \wedge q^{\prime} \vDash \bigwedge_{q^{\prime \prime} \in Q} \pi_{q^{\prime \prime}}$ and $q^{\prime} \notin Q$, which shows that $E\left(p^{\prime}\right) \subseteq E\left(q^{\prime}\right)$, which needed to be proved.

Part (2). Suppose $q \stackrel{a}{\longrightarrow} q^{\prime}$. We claim $\exists p^{\prime}: p \stackrel{a}{\Longrightarrow} p^{\prime}$. Suppose not. If $a \in V$ then for some $\left.\varphi \in E_{W B}\left(q^{\prime}\right), q \vDash<<a \gg\right\rangle$ and $\left.p \not \forall<<a \gg\right\rangle$. This implies $p \vDash \neg<<a \gg \varphi$ and $q \not \nvdash \neg\langle<a \gg\rangle \varphi$ which contradicts $E_{W B}(p) \subseteq E_{W B}(q)$. On the other hand, if $a=\tau$, then $p \stackrel{\tau}{\Longrightarrow}$ implies $p \vDash \varepsilon^{1} t t$. But since $q \stackrel{\tau}{\longrightarrow} q^{\prime}, q \not \models \varepsilon^{1} t t$ which contradicts $E(p) \subseteq E(q)$.

Now, we need to show that $\exists p^{\prime}: p \stackrel{a}{\Longrightarrow} p^{\prime} \wedge E\left(p^{\prime}\right) \subseteq E\left(q^{\prime}\right)$. Suppose there is no such $p^{\prime}$. Then $\forall p^{\prime}: p \stackrel{a}{\Longrightarrow} p^{\prime} \Longrightarrow E\left(p^{\prime}\right) \nsubseteq E E\left(q^{\prime}\right)$. Then for each weak $a$-derivative $p^{\prime \prime}$ of $p$, there exists a $\pi_{p^{\prime \prime}} \in E\left(p^{\prime \prime}\right)-E\left(q^{\prime}\right)$. Choose one such for each weak $a$-derivative $p^{\prime \prime}$ of $p$ and collect them in a set $\Pi$. Then we have $p \vDash[[a]](\bigvee \Pi)$, but $q \not \models[[a]](\bigvee \Pi)$, which contradicts $E(p) \subseteq E(q)$. Hence $\exists p^{\prime}: p \stackrel{a}{\Longrightarrow} p^{\prime} \wedge E\left(p^{\prime}\right) \subseteq E\left(q^{\prime}\right)$.

Lemma 19. $p \lesssim q$ implies $p \bigsqcup_{E} q$ i.e. $E(p) \subseteq E(q)$.

Proof. We need to show $p \lesssim q \Longrightarrow(p \vDash \pi \Longrightarrow q \vDash \pi)$. We prove this by induction on the structure of $\pi$

- $p \vDash \varphi$. Then $q \vDash \varphi$ follows from lemma 16.

- $p \vDash<<\hat{a} \gg>\pi, a \in$ Act. Then $\exists p^{\prime}: p \stackrel{\hat{a}}{\Longrightarrow} p^{\prime} \wedge p^{\prime} \vDash \pi$ and since $p \lesssim q, \exists q^{\prime}: q \stackrel{\hat{a}}{\Longrightarrow} q^{\prime} \wedge$ $\bar{p}^{\prime} \lesssim q^{\prime}$. Therefore, by induction hypothesis, $q^{\prime} \vDash \pi \Longrightarrow q \vDash<\langle\hat{a}>>\pi$.

- $p \vDash \varepsilon^{k} \pi$. Then $\forall p^{\prime}: p \stackrel{\tau^{j}}{\longrightarrow} p^{\prime} \wedge p^{\prime} \vDash \pi \Longrightarrow j<k$. Since $p \lesssim q$, we have, for every such

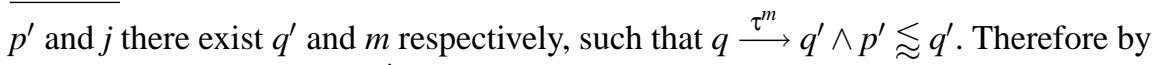
the induction hypothesis, $q^{\prime} \vDash \pi$. We claim that, $m \geq k$ is impossible. Suppose not; then $q \stackrel{\tau^{m}}{\longrightarrow} q^{\prime} \wedge p^{\prime} \lesssim q^{\prime}$ where $m \geq k$, for some $q^{\prime}$ and $m$. Then $\exists p^{\prime \prime}: p \stackrel{\tau^{j}}{\longrightarrow} p^{\prime \prime} \wedge p^{\prime \prime} \lesssim q^{\prime}$ for some $j \geq m \geq k$. But then $p \not \models \varepsilon^{k} \pi$ which is a contradiction. Hence $q \vDash \varepsilon^{k} \pi$.

- $p \vDash[[a]] \pi$. Then $\forall p^{\prime}: p \stackrel{a}{\Longrightarrow} p^{\prime} \Longrightarrow p^{\prime} \vDash \pi$. Since $p \lesssim q$, we have, for every such $p^{\prime}$, $\exists q^{\prime}: q \stackrel{\hat{a}}{\Longrightarrow} q^{\prime} \wedge p^{\prime} \lesssim q^{\prime}$. By the induction hypothesis $E\left(p^{\prime}\right) \subseteq E\left(q^{\prime}\right)$ and $q^{\prime} \vDash \pi$. We now claim that there does not exist any $q^{\prime}$ such that $q \stackrel{a}{\Longrightarrow} q^{\prime}$ and $q^{\prime} \not \models \pi$. Suppose the claim is false. Then we have $\exists q^{\prime}: q \stackrel{a}{\Longrightarrow} q^{\prime} \wedge q^{\prime} \not \models \pi$. This implies (by lemma 12) $\exists p^{\prime}: p \stackrel{a}{\Longrightarrow} p^{\prime} \wedge p^{\prime} \lesssim q^{\prime} \not \models \pi$. Again by induction hypothesis $E\left(p^{\prime}\right) \subseteq E\left(q^{\prime}\right)$ and hence $p^{\prime} \not \models \pi$. But this contradicts the assumption $p \vDash[[a]] \pi$. Hence the claim is false.

- $p \vDash \bigwedge_{i \in I} \pi_{i}$. Then $p \vDash \pi_{i}$ for all $i \in I$ and by the induction hypothesis, $q \vDash \pi_{i}$ for all

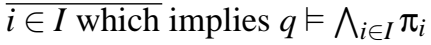

- $p \vDash \bigvee_{i \in I} \pi_{i}$. Then $p \vDash \pi_{i}$ for at least one $i \in I$ and by induction hypothesis it follows that $q \vDash \bigvee_{i \in I} \pi_{i}$.

The above proof has been presented in detail to highlight the steps for the operators at the higher level, especially the operator $\varepsilon^{k}$. Note that the proof would not go through if we allowed $\langle\langle\tau\rangle\rangle$ in the logic. 


\section{Efficiency Prebisimulation}

As we did for elaboration in the previous section, we now present a logical characterization for efficiency prebisimulation [2] using a logic which is more expressive than $L_{E}$ but not as expressive as $L_{S B}$.

The following formulation [2] gives a simple definition of the efficiency preorder. Closely related preorders have been defined independently by Milner in [10] and [11], though neither their algebraic nor logical characterizations have been presented.

Definition 20. A binary relation $R \subseteq P \times P$ is an efficiency prebisimulation (EP) iff for every $\langle p, q\rangle \in R, \alpha \in V, a \in$ Act the following conditions are satisfied.

$$
\begin{gathered}
p \stackrel{\alpha}{\longrightarrow} p^{\prime} \Longrightarrow \exists q^{\prime}: q \stackrel{\alpha}{\longrightarrow} q^{\prime} \wedge p^{\prime} R q^{\prime} \\
p \stackrel{\tau}{\longrightarrow} p^{\prime} \Longrightarrow p^{\prime} R q \vee\left(\exists q^{\prime}: q \stackrel{\tau}{\longrightarrow} q^{\prime} \wedge p^{\prime} R q^{\prime}\right) \\
q \stackrel{a}{\longrightarrow} q^{\prime} \Longrightarrow \exists p^{\prime}: p \stackrel{a}{\longrightarrow} p^{\prime} \wedge p^{\prime} R q^{\prime}
\end{gathered}
$$

We write $p \lesssim q$ if there exists an efficiency prebisimulation $R$ such that $p R q$.

Intuitively, $p \lesssim q$ means that for every execution sequence that $p$ may perform, it is possible to find a possibly shorter sequence that $q$ may perform with the same visible content, and conversely, for any sequence that $q$ may perform it is possible to find a possibly longer sequence that $p$ may perform with the same visible content. In general, both the preorders $\lesssim$ and $\lesssim$ represent comparisons between observationally equivalent processes and order them differently on the amount of internal computation they may perform. We refer to both preorders as efficiency-based preorders, but $\lesssim$ having been christened "efficiency preorder" earlier we continue to refer to it by the same name. We refer the reader to [2] and [1] for intuitively appealing examples and for the axiomatization of finite CCS processes.

In [3] it has been shown that $\sim \subset \lesssim \lesssim \lesssim \approx$ (where all the containments are strict). This suggests that we require a logic that is more expressive than $L_{E}$, but less than $L_{S B}$. This logic is very similar in structure to the logic for elaboration and is again defined in two levels with the lower level of weak formulae being the same.

Definition 21. The class $L_{E P}$ of efficiency prebisimulation formulae over Act is given by the following grammar. As before, we use $\alpha$ to denote a visible action and $a$ to denote any action.

$$
\begin{aligned}
& \varphi::=\left\langle<\alpha>\varphi\left|\bigwedge_{i \in I} \varphi_{i}\right| \neg \varphi\right. \\
& \pi::=\varphi|<\alpha>\pi|(\tau) \pi\left|\varepsilon^{k} \pi\right|[[a]] \pi\left|\bigwedge_{i \in I} \pi_{i}\right| \bigvee_{i \in I} \pi_{i}
\end{aligned}
$$

Note that the index set $I$ may be infinite. Also negation $(\neg)$ as in the case of $L_{E}$, is available only for weak formulae. The language $L_{E P}$ is the set of all formulae $\pi$ and the language $L_{W B}$ is the set of all weak formulae $\varphi$. Note that $L_{W B} \subseteq L_{E P}$. 
Definition 22. The satisfaction relation $\vDash \subseteq \mathbb{P} \times L_{E P}$ is defined recursively. As before, we omit those clauses which have been previously presented and restrict ourselves to defining the clauses for the new operators.

- $p \vDash<\alpha>\pi$ for $\alpha \in V$ if for some $p^{\prime} \in \mathbb{P}: p \stackrel{\alpha}{\longrightarrow} p^{\prime}$ and $p^{\prime} \vDash \pi$

- $p \vDash(\tau) \pi$ if $p \vDash \pi$ or for some $p^{\prime} \in \mathbb{P}: p \stackrel{\tau}{\longrightarrow} p^{\prime}$ and $p^{\prime} \vDash \pi$

$E P(p)=\left\{\pi \in L_{E P} \mid p \vDash \pi\right\}$ and $E P_{W B}(p)=\left\{\varphi \in L_{W B} \mid p \vDash \varphi\right\}$. Further, $p \sqsubseteq_{E P} q$ iff $E P(p) \subseteq E P(q)$, and $p={ }_{E P} q$ iff $E P(p)=E P(q)$. As before, $p \sqsubseteq_{E P_{W B}} q$ iff $E P_{W B}(p) \subseteq$ $E P_{W B}(q)$ and $p={ }_{E P_{W B}} q$ iff $E P_{W B}(p)=E P_{W B}(q)$.

As for the expressiveness of this language, notice that

- we allow the "strong possibility" modality (" $<\alpha>$ ") from $L_{S B}$, but neither negation nor "strong necessity" ("[ $\alpha]$ ").

- The operator " $\langle\tau\rangle$ " is replaced by the weaker prefix operator " $(\tau)$ ".

- The operator $\varepsilon^{k}$ in the formula $\varepsilon^{k} \pi$ excludes the possibility of a process being able to perform more than $k-1$ initial $\tau$ actions and reaching a state satisfying $\pi$.

- On the other hand, $p$ satisfying the formula $(\tau)^{k} \pi, k>0$ (obtained by prefixing $\pi$ by $k$ occurrences of $(\tau)$ ) asserts the existence of a $\tau^{j}$ derivative of $p$ (for some $j$, $0 \leq j \leq k)$ which satisfies $\pi$.

There are no formulae in $L_{E P}$ equivalent to the HML formula $\neg<\tau><\tau>\phi$. Even a statement such as " $p$ has a strong $\tau^{2}$-derivative that satisfies $\pi$ " can only be inferred if it is known that $p \not \models \pi, p \not \models(\tau) \pi$ and $p \vDash(\tau)^{2} \pi$.

We now proceed to give a proof outline of the characterization theorem. We begin with the following lemma which is clearly implied by the fact that every efficiency prebisimulation is a weak bisimulation and that $\sqsubseteq_{E P_{W B}}$ and $=_{E P_{W B}}$ coincide.

Lemma 23. $p \lesssim q \Longrightarrow p={ }_{E P_{W B}} q$ and hence $p \lesssim q \Longrightarrow p \sqsubseteq E P_{W B} q$.

Theorem 24. The characterization. $p \lesssim q$ iff $p \sqsubseteq E P q$.

We prove this theorem in two parts. We omit most of the routine details and concentrate on only the operators that have been newly introduced.

Lemma 25. $p \sqsubseteq_{E P} q$ implies $p \lesssim q$.

Proof. We show that $\sqsubseteq_{E P}$ is an efficiency prebisimulation. We prove the various parts viz. (5), (6) and (7) of definition (20), assuming $E P(p) \subseteq E P(q)$.

Part (5). Suppose $p \stackrel{\alpha}{\longrightarrow} p^{\prime}, \alpha \in V$. Then $p \vDash<\alpha>\pi$ for each $\pi \in E P\left(p^{\prime}\right)$. Clearly $q \vDash<\alpha>\pi$ and there exists $q^{\prime}$ such that $q \stackrel{\alpha}{\longrightarrow} q^{\prime}$ and $q^{\prime} \vDash \pi$. We need to show $\exists q^{\prime}$ : 
$q \stackrel{\alpha}{\longrightarrow} q^{\prime} \wedge E P\left(p^{\prime}\right) \subseteq E P\left(q^{\prime}\right)$. We use an argument that has now become routine viz. consider the set $Q=\left\{q^{\prime \prime} \mid q \stackrel{\alpha}{\longrightarrow} q^{\prime \prime} \wedge E P\left(p^{\prime}\right) \nsubseteq \subseteq E P\left(q^{\prime \prime}\right)\right\}$ and proceed as before.

Part (6). Suppose $p \stackrel{\tau}{\longrightarrow} p^{\prime}$, then for each $\pi \in E P\left(p^{\prime}\right)$ we have $p \vDash(\tau) \pi$ and $q \vDash(\tau) \pi$. Then if $q \not \models \pi$ there must exist a $q^{\prime}$ such that $q \stackrel{\tau}{\longrightarrow} q^{\prime}$ and $q^{\prime} \vDash \pi$. It then suffices to show that $\exists q^{\prime}: q \stackrel{\tau}{\longrightarrow} q^{\prime} \wedge E P\left(p^{\prime}\right) \subseteq E P\left(q^{\prime}\right)$. We may show this in a manner similar to that of Part 5.

Part (7). The proof of this part is similar to Part (2) of the proof of lemma 18.

Lemma 26. $p \lesssim q$ implies $p \sqsubseteq_{E P} q$.

Proof. Assume $p \lesssim q$. We again use induction on the structure of formulae. For any formula $\phi$ from $E P_{W B}$, lemma 23 assures us that $p \vDash \phi$ implies $q \vDash \phi$. Of the rest of the cases from the language $L_{E P}-L_{E P_{W B}}$ we consider only the following:

- $\underline{p \vDash<\alpha>\pi, \alpha \in V}$. Then for some $p^{\prime}$, we have $p \stackrel{\alpha}{\longrightarrow} p^{\prime}$ and $p^{\prime} \vDash \pi$. Hence $\exists q^{\prime}$ : $q \stackrel{\alpha}{\longrightarrow} q^{\prime} \wedge p^{\prime} \lesssim q^{\prime}$. By the induction hypothesis, $E P\left(p^{\prime}\right) \subseteq E P\left(q^{\prime}\right)$ and so we get $q \vDash<\alpha>\pi$.

- $p \vDash(\tau) \pi$. If $p \vDash \pi$ then by the induction hypothesis $q \vDash \pi$ and so it follows that $q \vDash(\tau) \pi$. On the other hand, if $p \not \models \pi$, then $\exists p^{\prime}: p \stackrel{\tau}{\longrightarrow} p^{\prime} \wedge p^{\prime} \vDash \pi$. From $p \lesssim q$ we have, either $p^{\prime} \lesssim q$ or $\exists q^{\prime}: q \stackrel{\tau}{\longrightarrow} q^{\prime} \wedge p^{\prime} \lesssim q^{\prime}$. If $p^{\prime} \lesssim q$ then $p^{\prime} \vDash \pi$ implies $q \vDash \pi$ by the induction hypothesis. If $p^{\prime} \& q$ then $p^{\prime} \vDash \pi$ implies $q^{\prime} \vDash \pi$ by the induction hypothesis and it follows that $q \vDash(\tau) \pi$.

- $p \models \varepsilon^{k} \pi, k>0$. This is again proved in a manner analogous to the corresponding proof for elaboration.

\section{Conclusion}

In the foregoing sections we have introduced two new operators into Hennessy-Milner Logic (HML) viz. $\varepsilon^{k}$ and $(\tau)$. We have shown and characterized preorders lying strictly between strong and weak bisimilarity using versions of HML and OHML with these new operators.

At the outset, we would first of all like to be convinced that their introduction into $L_{S B}$ does not in any way alter the expressive power of HML. It is easy to see from their semantics that these operators enjoy the following equivalences. For any formula $\pi$ in $L_{E}$ or $L_{E P}$, let $\tilde{\pi}$ denote the equivalent formula in $L_{S B}$. We then have

$$
\begin{aligned}
(\tau) \pi & \equiv \tilde{\pi} \vee<\tau>\tilde{\pi} \\
\varepsilon^{k} \pi & \equiv \bigwedge_{j \geq k}[\tau]^{j} \neg \tilde{\pi}
\end{aligned}
$$


While (8) is obvious from its semantics, it is fairly easy to derive (9) by noting that $p \not \models \varepsilon^{k} \pi$ precisely when $\exists p^{\prime}: p \stackrel{\tau^{j}}{\longrightarrow} p^{\prime} \wedge j \geq k \wedge\left(p^{\prime} \vDash \pi\right)$.

These operators also satisfy some other properties. For example, any formula $\pi \in$ $L_{E P}$ logically implies $(\tau) \pi$. And for any $k>0$ we have $(\tau)^{k} \pi \equiv \bigvee_{j \leq k}(\tau)^{j} \pi$. Further, $(\tau)^{k} \pi \Longrightarrow(\tau)^{k+1} \pi$. Similarly, in both $L_{E}$ and $L_{E P}$, for any $k>0$, we have $\varepsilon^{k} \pi \Longrightarrow$ $\varepsilon^{k+1} \pi$. For any set $S$ of positive integers, let $\inf (S)$ and $\sup (S)$ denote the minimum and maximum (provided it exists) elements of $S$ respectively. We then have the following identities.

$$
\begin{array}{llrl}
\bigwedge_{j \in S}(\tau)^{j} \pi \equiv(\tau)^{\inf (S)} \pi, & & \bigwedge_{j \in S} \varepsilon^{j} \pi \equiv \varepsilon^{\inf (S)} \pi \quad \text { and } \\
\bigvee_{j \in S}(\tau)^{j} \pi \equiv(\tau)^{\sup (S)} \pi, & \bigvee_{j \in S} \varepsilon^{j} \pi \equiv \varepsilon^{s u p(S)} \pi \text { if } S \text { is finite. }
\end{array}
$$

These properties enable us to follow the guiding principle that a preorder should be characterized by containment on sets of formulae.

If we were to extend the language to allow $\varepsilon^{0}$ as an operator, we would have that $p \vDash \varepsilon^{0} \pi$ implies $p \not \models \pi$. Then we would also be able to express the statement " $p$ diverges" by the formula $p \vDash \varepsilon^{0} \bigvee_{k>0} \varepsilon^{k} t t$ and the statement " $p$ can perform at least two $\tau$ actions" by $\varepsilon^{0} \varepsilon^{2} t$.

The results that we have presented in this paper offer interesting technical insights into the construction of logical characterizations of preorders guided by the principles enunciated in section 1. As far as we are aware, in the literature, there are no logical characterizations of behavioural relations that lie strictly between strong and weak bisimilarity.

In future work, we hope to study and understand more about these operators and explore their interactions with fixpoint operators within a modal $\mu$-calculus setting.

Acknowledgements We are grateful to Sanjiva Prasad and Astrid Kiehn for pointing out various simplifications and corrections in the design of our logics, and for their patient reading and other constructive suggestions. In particular Sanjiva Prasad also pointed out that $\varepsilon^{0}$ would allow the full power of negation. We also thank Jamshid Bahgerzadeh for his valuable inputs and suggestions.

This work was partly supported by a research grant from MHRD, Governement of India, under the Scheme of Research and Development in Technical Education (File No. F.261/2002-TS.V dated 19 March 2002).

\section{References}

1. S. Arun-Kumar and M. Hennessy: An Efficiency Preorder for Processes. Proceedings Theoretical Aspects of Computer Software, Sendai 1991, Lecture Notes in Computer Science 526, 152-175, Springer-Verlag 1991.

2. S. Arun-Kumar and M.Hennessy: An Efficiency Preorder for Processes. Acta Informatica, 29, 737-760, Springer-Verlag, 1992. 
3. S. Arun-Kumar and V. Natarajan: Conformance: A Precongruence Close to Bisimilarity. Proceedings Structures in Concurrency Theory, Springer Workshops in Computer Science Series, 1995.

4. F. Corradini and R. Gorrieri and M. Rocetti: Performance Preorder and Competitive Equivalence. Acta Informatica, 34, 805-835, 1997.

5. M. Hennessy and R. Milner: Algebraic Laws for Nondeterminism and Concurrency. Journal of ACM, 32, 137-161, 1985.

6. Kamal Jain and S. Arun-Kumar: Testing Processes for Efficiency. Proceedings Fondations of Software Technology \& Theoretical Computer Science 16, Lecture Notes in Computer Science 1180, 100-110, Springer-Verlag, 1996.

7. L. Jenner and W. Vogler: Comparing the Efficiency of Asynchronous Systems. Technical Report 1998-3, Universität Augsburg, December 1998.

8. G. Luettgen and W. Vogler: A Faster than relation for Asynchronous Processes. Proceedings CONCUR 2001, Lecture Notes in Computer Science 2154, 262-276,

9. R. Milner: Communication and Concurrency, Prentice-Hall International, 1989.

10. R. Milner: Contractions, Handwritten notes, 1990.

11. R. Milner: Expansions. Handwritten notes, 1990

12. C. Stirling: Modal and Temporal Properties of Processes, Springer-Verlag 2001.

13. V. Natarajan and R. Cleaveland: An Algebraic Theory of Process Efficiency. Proceedings Logic in Computer Science '96, IEEE Computer Society Press 1996.

14. R. J. van Glabeek: The Linear Time - Branching Time Spectrum I. The Semantics of Concrete, Sequential Processes. In Handbook of Process Algebra, (eds.) J. A. Bergstra, A. Ponse and S. A. Smolka, Elsevier Science B. V., Netherlands., 2001. 